\title{
Common Carotid Intima-Media Thickness as an Indicator of Atherosclerosis at Other Sites of the Carotid Artery
}

\author{
The Rotterdam Study
}

\author{
MICHIEL L. BOTS, MD, PhD, A. HOFMAN, MD, PhD, PAUlUS T. V. M. DE JONG, MD, PhD, \\ AND DIEDERICK E. GROBBEE, MD, PHD
}

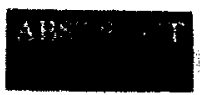

To evaluate the association between common carotid intima-media thickness and noninvasively assessed atherosclerosis elsewhere in the carotid artery, we used data from the Rotterdam Study, a single-center population-based, prospective follow-up study among 7983 subjects aged 55 years or older. Baseline measurements included ultrasonographic evaluation of the vessel wall characteristics of the carotid arteries, that is, assessment of the presence of atherosclerotic lesions, measurement of common carotid intima-media thickness, and determination of the presence of hemodynamically significant stenosis. The present analysis is based on data obtained from the first 1000 participants. The results were adjusted for age and gender. An increase of 1 standard deviation in common carotid intima-media thickness $(0.19 \mathrm{~mm})$ dunbled the risk of plaques in the carotid bifurcation: odds ratio 2.0 (95\% confidence interval $(\mathrm{Cl}): 1.6,2.4)$. For hemodynamically significant stenosis of the right internal carotid artery $(\geqslant 50 \%)$, a 1.4 (95\% CI: 1.0, 2.0) increased risk was found. The risk of atherosclerotic plaques in the bifurcation in subjects with an intima-media thickness above $0.89 \mathrm{~mm}$ (upper quintile) relative to those with an intima-media thickness below $0.63 \mathrm{~mm}$ (lowest quintile) was $6.0195 \% \mathrm{Cl}$ : $3.5,10.2)$. For intemal carotid artery stenosis, a relative risk of $2.8(95 \% \mathrm{Cl} 0.5,15.7)$ was found. The findings of the present study provide evidence that increased common carotid intima-media thickness indicates atherosclerosis at other sites of the carotid artery. This study lends support to the view that noninvasively assessed increased intima-media thickness of the distal common carotid artery is an indicator of generalized atherosclerosis. Ann Epidemiol 1996;6:147-153.

KEY WORDS: Atherosclerosis, ultrasonography, carotid stenosis, cardiovascular disease, population based.

\section{INTRODUCTION}

High-resolution B-mode ultrasonography enables accurate assessment of vessel wall characteristics of the carotid arteries in populations at large $(1,2)$. This technique facilitates evaluation of the lumen diameter, the intima-media thickness, and the presence and extent of plaques of the carotid artery (3-7). B-mode combined with Doppler spectral analysis (duplex) can be used to assess hemodynamically signifcant stenosis of the carotid artery, which is indicative of advanced atherosclerotic vessel wall disease (8). Furthermore, the atherosclerotic process can be studied in an earlier phase through measurement of intima-media thickness of

From the Department of Epidemiology and Biostatistics, Erasmus University Medical School (M.L.B., A.H., D.E.G.), and the Department of Ophthalmology, University Hospital Rotterdam (P.T.V.M.d.J.), Rotterdam, the Netherlands.

Address reprint requests to: Diederick E. Grobbee, MD, PhD, Department of Epidemiology and Biostatistics, Erasmus University Medical School, PO Box 1738, 3000 DR Rotterdam, the Netherlands.

Received August 23, 1994; revised May 10, 1995; accepted December 15, 1995. the common carotid artery. As the common carotid artery remains relatively spared from development of severe atherosclerosis, the applicability of measurement of common carotid intima-media thickness with respect to the atherosclerotic process is conditional on the degree to which increased common carotid intima-media thickness reflects atherosclerotic vessel wall disease in other atteries that are at high risk of atherosclerosis. It is important to answer the question to what extent increased intima-media thickness of the common carotid artery may be regarded as an indicator of generalized atherosclerosis. A limited number of studies on this issue were recently performed. Two large population-based studies in the United States in middle-aged and elderly subjects showed a positive association between common carotid intima-media thickness and atherosclerosis in the carotid bifurcation (9) and in the internal carotid artery $(9,10)$. We earlier reported on the positive assuciation between common carotid intima-media thickness and atherosclerosis in the abdominal aorta (11) and the arteries of the lower extremities among an older white population in the Netherlands (12).

In this article we report on the association of noninva- 
sively assessed common carotid intima-media thickness and atherosclerotic abnormalities in the carotid bifurcation and internal carotid artery among the first 1000 participants in the Rotterdam Study.

\section{METHODS}

\section{Population}

The Rotterdam Study is a single-center, prospective, follow-up study of 7983 men and women aged 55 years or older, investigating the incidence of and risk factors for chronic disabling diseases (13). The study was approved by the Medical Ethics Committee of Erasmus University and written informed consent was obtained from all participants. All inhabitants 55 years or older living at one point in time in the Rotterdam suburb of Ommoord were invited to participate. The study comprised an extensive home interview, followed by two visits at the Rotterdam study research center for clinical examinations. Baseline examinations were performed from March 1990 to July 1993. The participation rate of those invited was $78 \%$.

\section{Ultrasonography of the Carotid Arteries}

To measure carotid intima-media thickness, ultrasonography of both carotid arteries was performed with a $7.5-\mathrm{MHz}$ linear-array transducer (ATL UltraMark IV, Advanced Technology Laboratories, Bothell, WA). The actual obtained axial resolution is around 0.3 to $0.5 \mathrm{~mm}$, whereas the manufacturer's specification provides an estimate of the axial resolution of $0.5 \mathrm{~mm}$ (14). On a longitudinal twodimensional ultrasound image of the carotid artery, the anterior (near) and posterior (far) wall of the carotid artery were displayed as two bright white lines separated by a hypoechogenic space (15). The distance from the leading edge of the first bright line of the far wall (lumen-intima interface) to the leading edge of the second bright line (media-adventitia interface) indicated the intima-media thickness $(15,16)$. Following the Rotterdam Study ultrasound protocol, a careful search was performed for the lumenintima interface and the media-adventitia interface of the far wall of the distal common carotid artery $(6,12)$. When an optimal longitudinal image was obtained, it was frozen on the $R$ wave of the electrocardiogram and stored on videotape. This procedure was repeated three times for both sides. The actual measurements of intima-media thickness were performed off-line. From the videotape, the frozen images were digitized and displayed on the screen of a personal computer using additional dedicated software. This procedure was described in detail elsewhere $(6,16)$. In short, with a cursor the interfaces of the distal common carotid artery were marked over a length of $10 \mathrm{~mm}$. The beginning of the dilatation of the distal common carotid artery served as a reference point for the start of the measurement. The average of the intima-media thickness of each of the three frozen images was calculated. For each subject a mean far wall intima-media thickness ((left + right)/2) was taken as a measure for current wall thickness. Results from a reproducibility study of intima-media thickness measurements among 80 participants in the Rotterdam Study who underwent a second ultrasound scan of both carotid arteries within 3 months of the first scan were published elsewhere (17). In short, mean differences (standard deviation (SD)) in far wall intima-media thickness of the common carotid artery between paired measurements of sonographers, readers, and visits were $-0.005 \mathrm{~mm}(0.09), 0.060 \mathrm{~mm}(0.05$, and $-0.033 \mathrm{~mm}(0.12)$, respectively.

The common carotid artery and the carotid bifurcation were evaluated off-line (from tapes) for the presence (yes/ no) of atherosclerotic lesions on both the near and the far wall of the carotid artery. Plaques were defined as a focal widening relative to adjacent segments, with protrusion into the lumen composed of either only calcified deposits or a combination of calcification and noncalcified material. No attempt was made to quantify the size or extent of the lesions. A reproducibility study among 166 participants on the assessment of plaques in the carotid bifurcation revealed a $\kappa$ of 0.59 for the left carotid artery, of 0.65 for the right carotid artery, and of 0.60 for plaques in either side. The findings were statistically significant $(p<0.001)$ and indicate a moderate agreement.

Hemodynamically significant stenosis of the carotid artery was ultrasonographically assessed using a $7.5-\mathrm{MHz} \mathrm{sec}$ tor transducer in combination with a $5-\mathrm{MHz}$ pulsed Doppler (18). For reasons of feasibility, only the right carotid artery was evaluated for stenosis. Interpretation of velocity profiles was done on-line according to standard criteria based on the systolic peak velocity, the end-diastolic velocity, and presence of spectral broadening (19). The right internal carotid artery was categorized as being normal $(0 \%$ reduction of lumen diameter) or having minimal lesions (1 to $15 \%$ reduction), moderate stenosis (16 to $49 \%$ reduction), or severe stenosis ( $\geqslant 50 \%$ reduction).

\section{Data Analysis}

The present analysis is based on findings in the first 1000 participants of the Rotterdam Study. In 12 subjects ultrasonography of the carotid arteries could not be performed due to technical or logistical reasons. In 19 subjects, extreme tortuosity of the right internal carotid artery prevented reliable measurements of stenosis. Data on atherosclerotic lesions in the carotid bifurcation were not available for 140 subjects.

For 31 subjects measurement of common carotid intimamedia thickness at either the left or the right carotid artery could not be performed from the stored images because of 
poor visualization. In these subjects the estimate of common carotid intima-media thickness was based on the measurement of the side for which a value was available.

The direction and magnitude of the association between common carotid intima-media thickness and atherosclerosis at other sites of the carotid artery were estimated using logistic regression analysis. Multiple logistic regression analysis was used to adjust for differences in age and gender. Results are presented with a $95 \%$ confidence interval (CI). Analyses with common carotid intima-media thickness as a continuous variable and categorized in quintiles were performed. Separate analyses were done to assess the association of left and right carotid intima-media thickness with ipsilateral and contralateral atherosclerotic lesions.

\section{RESULTS}

The study population consisted of 600 women with a mean age of 68.9 years (SD: 8.0 ) and 388 men with a mean age of 68.6 years (SD: 7.7). The distribution of common carotid intima-media thickness is given in Figure 1 for men and women and the left and right sides. The mean common carotid intima-media thickness was $0.76 \mathrm{~mm}$ (SD: 0.19) for wornen and $0.80 \mathrm{~mm}$ (SD: 0.19) for men. The prevalence of plaques in the common carotid artery and in the carotid bifurcation in women was $11.3 \%$ and $48.1 \%$, respectively. For men, prevalences of $18.3 \%$ and $56.9 \%$ were found. Hemodynamically significant stenosis $(\geqslant 50 \%)$ was found in $0.7 \%$ of the women and $2.6 \%$ of the men.

An increase of 1 SD of common carotid intima-media thickness $(0.19 \mathrm{~mm})$ doubled the risk of plaques in the carotid bifurcation: odds ratio $2.0(95 \% \mathrm{CI}: 1.6,2.4)$. A gradual increased risk of atherosclerotic lesions in the carotid bifurcation was found with increasing common carotid intimamedia thickness (Table 1). The risk of atherosclerotic plaques in the bifurcation in subjects with an intima-media thickness above $0.89 \mathrm{~mm}$ (upper quintile) relative to those with an intima-media thickness below $0.63 \mathrm{~mm}$ (lowest quintile) was 6.0 ( $95 \% \mathrm{Cl}: 3.5,10.2$ ). The findings for men and women were $5.0(95 \% \mathrm{CI}: 2.1,11.7)$ and $7.1(95 \% \mathrm{CI}$ : $3.5,14.4)$, respectively.

An increase in common carotid intima-media thickness of $1 \mathrm{SD}$ was associated with a $40 \%$ increased risk of hemodynamically significant stenosis of the right internal carotid artery ( $\geqslant 50 \%$ lumen reduction): odds ratio $1.4(95 \% \mathrm{CI}$ : $1.0,2.0)$. For internal carotid artery stenosis an odds ratio of 2.8 (95\% CI: $0.5,15.7)$ was found comparing the upper and the lower intima-media thickness quintile.

Findings from analyses on the association between left and right common carotid intima-media thickness and ipsilateral and contralateral atherosclerotic lesions are presented in Table 1 . There are no clear differences in direction of the associations, although the strength of the associations do vary. Compared to the analyses in which both left and right intima-media thickness measurements were averaged, the magnitude of the association for left and right intimamedia thickness separately was considerably reduced (see Table 1). The odds ratio of stenosis of the right internal carotid artery associated with a SD increase in left common carotid intima-media thickness was $1.5(95 \% \mathrm{CI}: 1,1,1.9)$. For the right common carotid intima-media thickness, an odds ratio of $1.1(95 \% \mathrm{Cl}: 0.7,1.6)$ was found.

Figure 2 indicates that the presence of atherosclerotic lesions in the carotid bifurcation gradually increases with increasing common carotid intima-media thickness without clear evidence for the presence of a cutoff point. Among subjects with a common carotid intima-media thickness of $1.0 \mathrm{~mm}$ or larger (i.e., $9 \%$ of the study population), the prevalence of atherosclerotic lesions in the carotid bifurcation was $70 \%$ or higher. Similarly, the presence of atherosclerotic lesions in the near and far walls of the common carotid artery gradually increased with increasing far wall common carotid intima-media thickness (see Figure 2).

\section{DISCUSSION}

The findings in this population-based study of elderly subjects indicate that an increased common carotid intimamedia thickness is significantly associated with atherosclerotic lesions at other sites of the carotid artery, such as the carotid bifurcation and the right internal carotid artery. Associations were stronger when information on intimamedia thickness from both the left and the right side was combined.

The nature of the intima-media thickness is still a point of discussion, that is, whether increased common carotid intima-media thickness itself reflects atherosclerosis. Increased common carotid intima-media thickness may merely reflect an adaptive response of the vessel wall to changes in shear stress and tensile stress (20). Atherosclerosis is viewed as a disorder that is restricted to the intimal layer of the arterial vessel wall (21), and ultrasound imaging can not discriminate between the intima layer and the media layer of the vessel wall. Yet, several studies compared the precise location of the interfaces as seen with high-resolution B-mode ultrasound with the histologic layers of the arterial wall. Results from these studies showed that the distance between the lumen-intima interface and the mediaadventitia interface at the far wall as seen with ultrasound truly reflects intima-media thickness $(15,22,23)$. Unfortunately, the issue at what level intima-media thickness is predominantly determined by an atherosclerotic component was not addressed. From other studies it seems likely that above a certain level (e.g., $1.0 \mathrm{~mm}$ ), intima-media thickness is indicative of atherosclerosis $(24,25)$. Our findings of a gradual positive association between common carotid 

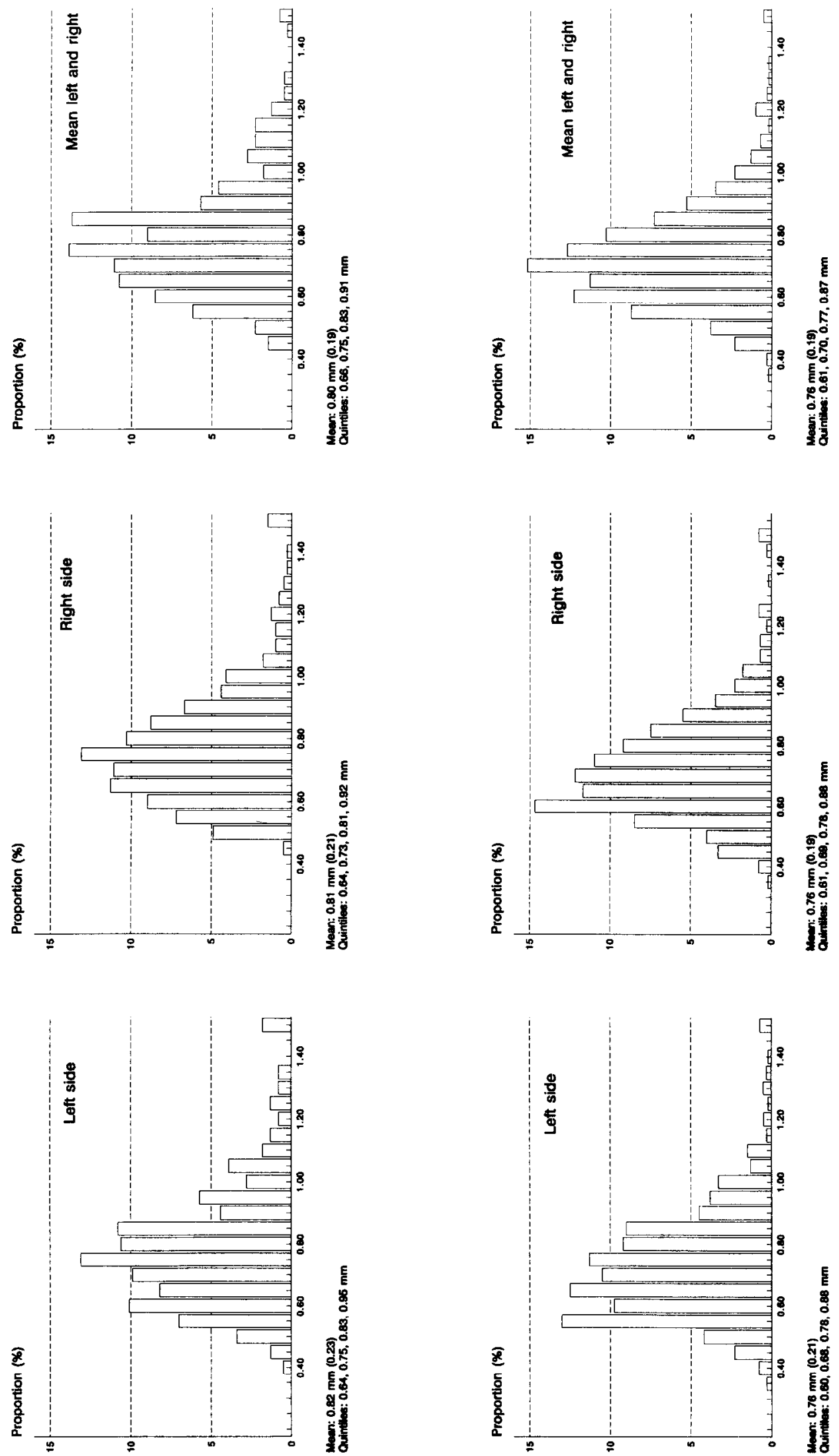

FIGURE 1. Distribution of common carotid intima-media thickness for men (top row) and women (bottom row) for the left and right sides. 
TABLE 1. The association (odds ratio $(95 \% \mathrm{CI})$ between left and right common carotid intima-media thickness and ipsilateral and contralateral atherosclerotic lesions in the carotid bifurcation ${ }^{a}$

\begin{tabular}{|c|c|c|c|c|c|}
\hline & \multicolumn{5}{|c|}{ Quintile of common carotid intima-media thickness } \\
\hline & Q1 & Q2 & Q3 & Q4 & Q5 \\
\hline Right CCA vs. right BIF & 1.0 & $1.1(0.7,1.9)$ & $1.5(0.9,2.6)$ & $1.9(1.1,3.1)$ & $3.0(1.8,5.0)$ \\
\hline Right CCA vs. left BIF & 1.0 & $1.5(0.9,2.5)$ & $1.3(0.7,2.2)$ & $2.0(1.2,3.4)$ & $2.4(1.4,4.1)$ \\
\hline Left $\mathrm{CCA}$ vs. right $\mathrm{BIF}$ & 1.0 & $1.6(1.0,2.7)$ & $1.3(0.8,2.2)$ & $2.0(1.2,3.4)$ & $3.8(2.2,6.4)$ \\
\hline Left CCA vs. left BIF & 1.0 & $1.0(0.6,1.7)$ & $1.3(0.8,2.2)$ & $1.3(0.8,2.1)$ & $2.6(1.5,4.4)$ \\
\hline Left + right CCA vs. right $\mathrm{BIF}$ & 1.0 & $1.5(0.9,2.6)$ & $1.5(0.9,2.5)$ & $1.9(1.1,3.3)$ & $4.6(2.7,7.9)$ \\
\hline Left + right CCA vs. left BIF & 1.0 & $1.1(0.7,1.9)$ & $1.4(0.8,2.2)$ & $1.4(0.8,2.4)$ & $3.4(2.0,5.7)$ \\
\hline Left + right CCA vs. left + right BIF & 1.0 & $1.5(0.9,2.4)$ & $1.7(1.0,2.7)$ & $2.0(1.2,3.3)$ & $6.0(3.5,10.2)$ \\
\hline
\end{tabular}

"Adjusted for age and gender.

C.CA, common carotid artery; BIF, carotid bifurcation.

intima-media thickness and plaques at the far wall of the common carotid artery (see Figure 2), however, do not lend support to the presence of a clear cutoff level.

Evidence to indicate that intima-media thickness is a measure of atherosclerosis comes from several crosssectional studies which consistently showed that increased intima-media thickness of the common carotid artery is associated with unfavorable levels of cardiovascular risk factors $(4-6,26-28)$. The observation that progression of common carotid intima-media thickness over time is associated with risk factors for atherosclerosis (29), and the results from a very limited number of trials on the efficacy of lipidlowering drugs to reduce progression of intima-media thickness, which showed a reduced progression of (common) carotid intima-media thickness in the treated group compared to the placebo group (30-33), further strengthen the view that common carotid intima-media thickness is a measure of atherosclerosis.

Recent findings from the Cardiovascular Health Study showed a strong association between maximum common carotid intima-media thickness and severity of internal carotid atherosclerosis (10). Results from the Atherosclerosis

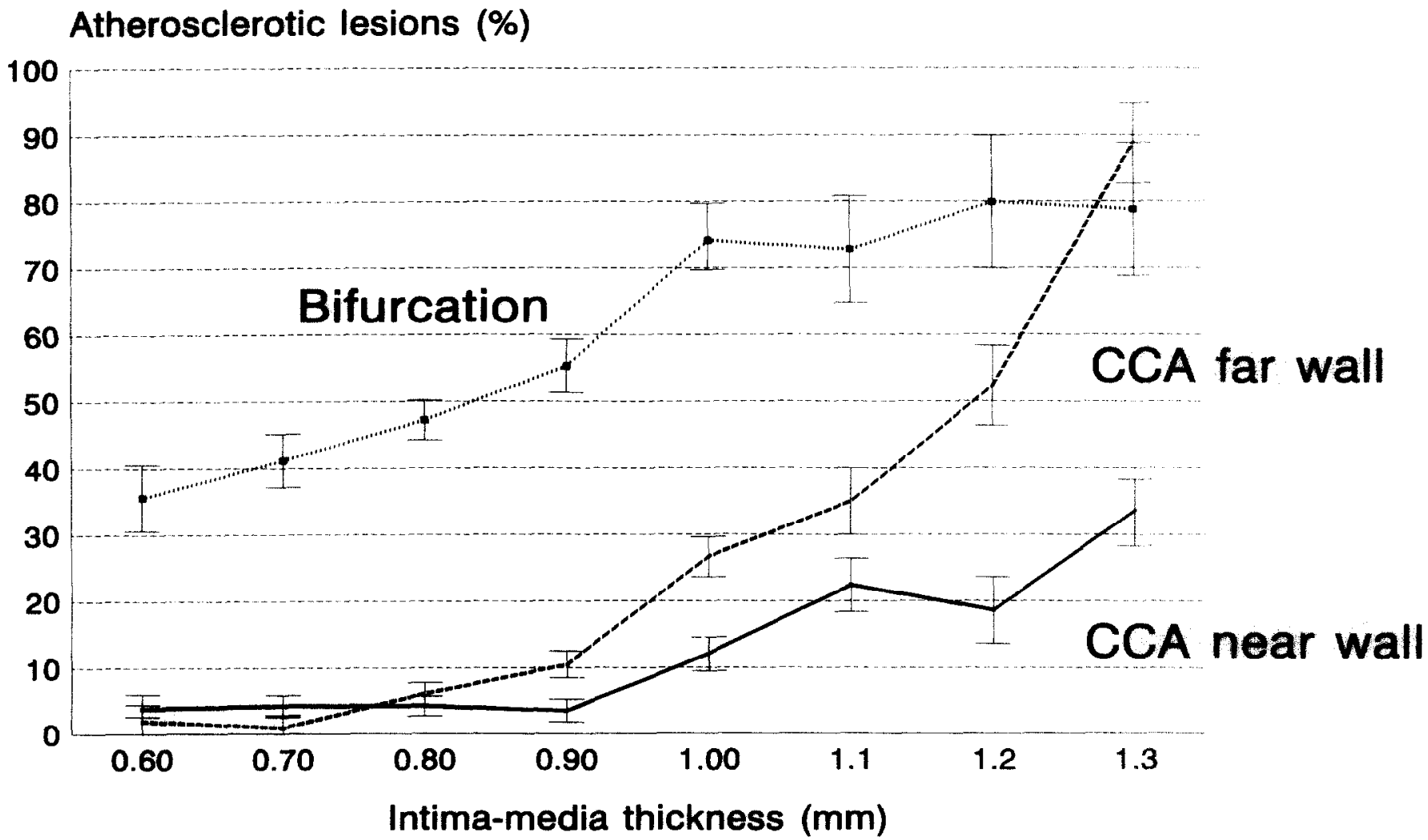

FIGURE 2. Common carotid intima-media thickness and prevalence (standard error) of atherosclerotic lesions in the carotid bifurcation and the near and far walls of the distal common carotid artery (CCA). 
Risk In Communities (ARIC) study demonstrated positive, but modest associations between common carotid intimamedia thickness and intima-media thickness at other sites of the carotid artery (9). In earlier reports, we showed that an increased common carotid intima-media thickness is associated with atherosclerosis at other sites, such as in the abdominal aorta (11) and in the arteries of the lower extremities (12). Prospective findings from the Kuopio Ischemic Heart Disease Risk Factor study showed that an increased common carotid intima-media thickness predicts myocardial infarction (34). These results, including the present findings, lend support to the view that noninvasively assessed intima-media thickness of the distal common carotid artery may provide an indicator of generalized atherosclerosis and cardiovascular risk (35).

In the present study the magnitude of the association between ipsilateral and contralateral abnormalities with left and right common carotid intima-media thickness was considerably reduced compared to the association found for the average of left and right far wall common carotid intimamedia thicknesses. Most of the variability in common carotid intima-media thickness can be attributed to the presence of focal atherosclerotic abnormalities. Combining the information from both the left and the right carotid artery probably results in an estimate for carotid atherosclerosis with enhanced precision, and subsequently strong associations are found. The question as to whether the far wall intima-media thickness of the common carotid artery is the most appropriate marker for studies on determinants of change in intima-media thickness over time, and on intimamedia thickness as a predictor of morbidity and mortality can not be satisfactorily answered, yet. Such information is urgently awaited.

Ultrasonographic assessment of carotid intima-media thickness provides a promising approach to study the presence and progression of atherosclerosis. Through measurement of intima-media thickness, the atherosclerotic process can be studied in an early phase as well as in an advanced phase in nonhospitalized and asymptomatic subjects (36). It enables evaluation of the natural history and the determinants of the presence and progression of atherosclerosis in population-based studies. However, although likely, conclusive evidence of a causal link between increased common carotid intima-media thickness and subsequent cardiovascular events is still awaited. The same applies to data regarding changes, for example, reductions in intima-media thickness and subsequent changes in cardiovascular risk. There is a clear need for these data $(37,38)$. Given the attention presently paid to intima-media thickness and its meaning in basic, clinical, and epidemiologic studies, such data will become available soon.

In conclusion, the findings of the present study provide evidence that increased common carotid intima-media thickness is an indicator of atherosclerosis at other sites of the carotid artery. These findings further support the view that noninvasively assessed intima-media thickness of the distal common carotid artery may provide an indication of generalized atherosclerosis.

\section{REFERENCES}

1. Salonen R, Haapanen A, Salonen JT. Measurement of intima-media thickness of common carotid arteries with high resolution B-mode ultrasonography: Inter- and intra-observer variability, Ultrasound Med Biol. 1991;17:225-230.

2. O'Leary DH, Polak JF, Wolfson SK, et al, on behalf of the CHS Collaborative Group. Use of sonography to evaluate carotid atherosclerosis in the elderly. The Cardiovascular Health Study, Stroke. 1991;22:1155-1163.

3. Heiss G, Sharett AR, Barnes R, Chambless LE, Szklo M, Alzola C, the ARIC Investigators. Carotid atherosclerosis measured by B-mode ultrasound in populations: Associations with cardiovascular risk factors in the ARIC study, Am J Epidemiol. 1991;134:250-256.

4. Wendelhag $\mathrm{I}$, Olov G, Wikstrand J. Arterial wall thickness in familial hypercholesterolemia. Ultrasound measurements of intima-media thickness in the common carotid artery, Arterioscler Thromb. 1992; 12:70-77.

5. Salonen R, Seppänen K, Rauramaa R, Salonen JT. Prevalence of carotid atherosclerosis and serum cholesterol levels in eastern Finland, Arteriosclerosis. 1988;8:788-792.

6. Bots ML, Hofman A, Bruyn AM, de Jong PTVM, Grobbee DE. Isolated systolic hypertension and vessel wall thickness of the carotid artery: The Rotterdam Study, Arterioscler Thromb. 1993;13:64-69.

7. Psaty BM, Furberg CD, Kuller LH, et al. Isolated systolic hypertension and subclinical cardiovascular disease in the elderly. Initial findings from the Cardiovascular Health Study, JAMA. 1992;268:1287-1291.

8. Feussner JR, Matchar DB. When and how to study the carotid arteries, Ann Intern Med. 1988;109:805-818.

9. Howard G, Burke GL, Evans GW, et al, for the ARIC Investigators. Relations of intimal-medial thickness among sites within the carotid artery as evaluated by B-mode ultrasound, Stroke. 1994;25:1581-1587.

10. Polak JF, O'Leary DH, Kronmal RA, et al. Sonographic evaluation of the carotid artery atherosclerosis in the elderly: Relationship of disease severity to stroke and transient ischemic attack, Radiology. 1993; 188:363-370.

11. Bots ML, Witteman JCM, Grobbee DE. Carotid intima-media wall thickness in elderly women with and without atherosclerosis of the abdominal aorta, Atherosclerosis. 1993;102:99-105.

12. Bots ML, Hofman A, Grobhee DE. Common carotid intima-media thickness and lower extremity arterial atherosclerosis. The Rotterdam Study, Arterioscler Thromb. 1994;14:1885-1891.

13. Hofman A, Grobbee DE, de Jong PTVM, Vandenouweland FA. Determinants of disease and disability in the elderly. The Rotterdam Elderly Study, Eur J Epidemiol. 1991;7:403-422.

14. Advanced Technology Laboratories. $7.5 \mathrm{MHz}$ High Frequency Linear Array Specification. ATL, Inc. Bothell, WA, 1988.

15. Pignoli P, Tremoli E, Poli A, Oreste P, Paoletti R. Intimal plus medial thickness of the arterial wall: A direct measurement with ultrasound imaging, Circulation. 1986;74:1399-1406.

16. Wendelhag I, Gustavsson T, Suurküla M, Berglund G, Wikstrand J. Ultrasound measurement of wall thickness in the carotid artery: Fundamental principles, and description of a computerized analyzing system, Clin Physiol. 1991;11:565-577.

17. Bots ML, Mulder PGH, Hofman A, van Es GA, Grobbee DE. Reproducibility of carotid vessel wall thickness measurements. The Rotterdam Study, J Clin Epidemiol. 1994;47:921-930.

18. Bots ML, Breslau PJ, Briët E, et al. Cardiovascular determinants of 
carotid artery disease: The Rotterdam Elderly Study, Hypertension. 1992;19:717-720.

19. Taylor DC, Strandness DE. Carotid artery duplex scanning, J Clin Uitrasound. 1987;15:635-644.

20. Glagov S, Vito R, Giddens DP, Zarins CK. Micro-architecture and composition of artery walls: Relationships to location, diameter and the distribution of mechanical stress, J Hypertens. 1992;10(suppl 6): S1010-S104.

21. Stary HC, Blankenhorn DH, Chandler B, et al. A definition of the intima of human arteries and of its atherosclerosis-prone regions, Arterioscler Thromb. 1992;12:120-134.

22. Wong M, Edelstein J, Wollman J, Bond MG. Ultrasonic-pathological comparison of the human arterial wall. Verification of intima-media thickness, Arterioscler Thromb. 1993;13:482-486.

23. Pignoli P. Ultrasound B-mode imaging for artery wall thickness measurement, Atheroscler Rev. 1984:12:177-184.

24. Bond MG, Ball M. Assessment of Ultrasound B-Mode Imaging for Detection and Quantification of Atherosclerotic Lesions in Arteries of Animals. NHLBI Nol-HV-12916. Bethesda, MD: National Heart, Lung, and Blood Institute; 1986.

25. Ricotta JJ, Bryan FA, Bond MG, et al. Multicenter validation study of real time (B-mode) ultrasound, arteriography, and pathologic examination, J Vasc Surg. 1987;6:512-520.

26. Haapanen A, Koskenvuo M, Kaprio J, Kesåaniemi YA, Heikkilå K. Carotid arteriosclerosis in identical twins discordant for cigarette smoking, Circulation. 1989;80:10-16.

27. Poli A, Tremoli E, Colombo A, Sitrori M, Pignoli P. Ultrasonographic measuremenr of the common carotid artery wall thickness in hypercholesterolemic patients, Atherosclerosis. 1988;80:253-261.

28. Bonithon-Kopp C, Scarabin P, Taquet A, Touboul P, Malmejac A,
Guize L. Risk factors for early carotid atherosclerosis in middle-aged French women, Arterioscler Thromb. 1991;11:966-972.

29. Salonen R, Salonen JT. Progression of carotid atherosclerosis and its determinants: A population-based ultrasonography study, Atherosclerosis. $1990 ; 81: 33-40$

30. Blankenhorn DH, Selzer RH, Crawford DW, et al. Beneficial effects of colestipol-niacin thickness on the common carotid artery, Circulation. 1993;88:20-28.

31. Furberg CD, Adams HP, Applegate WB, et al. Effect of lovastatin on early carotid atherosclerosis and cardiovascular events, Circulation. 1994;90:1679-1687.

32. Crouse JR, Byington RP, Bond MG, et al. Pravastatin, lipids and atherosclerosis in the carotid arteries: Design features of a clinical trial with carotid atherosclerosis outcome, Controlled Clin Trials. 1992; 13:495-506.

33. Salonen JT, Salonen R. Risk factors for carotid and femoral atherosclerosis in hypercholesterolemic men, J Intern Med. 1994;236:561-566.

34. Salonen JT, Salonen R. Ultrasonographically assessed carotid mor phology and the risk of coronary heart disease, Arterioscler Thromb. $1991 ; 11: 1245-1249$

35. Grobbee DE, Bots ML. Carotid artery intima-media thickness as an indicator of generalized atherosclerosis, ] Intern Med. 1994:236:567573.

36. Joensuu T, Salonen R, Winblad I, Korpela H, Salonen JT. Determinants of femoral and carotid artery atherosclerosis, J Intern Med. 1994 236:799-784.

37. Wikstrand J, Wiklund $O$. Frontiers in cardiovascular science. Quantitative measurements of atherosclerotic manifestations in humans, Ar terioscler Thromb. 1992;12:114-119.

38. Pearson TA, Heiss G. Atherosclerosis. Quantitative imaging, risk factors, prevalence and change, Circulation. 1993;87(suppl II):1-80. 